\title{
Absence of conventional quantum phase transitions in itinerant systems with disorder
}

\author{
V. Dobrosavljević ${ }^{1}$ and E. Miranda ${ }^{1,2}$ \\ ${ }^{1}$ Department of Physics and National High Magnetic Field Laboratory, \\ Florida State University, Tallahassee, Florida 32306, USA. \\ ${ }^{2}$ Instituto de Física Gleb Wataghin, Unicamp, Caixa Postal 6165, Campinas, SP, CEP 13083-970, Brazil.
}

(Dated: 24th June 2018)

\begin{abstract}
Effects of disorder are examined in itinerant systems close to quantum critical points. We argue that spin fluctuations associated with the long-range part of the RKKY interactions generically induce non-Ohmic dissipation due to rare disorder configurations. This dissipative mechanism is found to destabilize quantum Griffiths phase behavior in itinerant systems with arbitrary symmetry of the order parameter, leading to the formation of a "cluster glass" phase preceding uniform ordering.
\end{abstract}

PACS numbers: 75.40.-s, 71.10.Hf, 71.27.+a, 75.10.Nr

The effects of quenched disorder seem to be especially important near quantum phase transitions and lead to new classes of phenomena dominated by rare disorder configurations. At present, this behavior is rather well understood in insulating disordered magnets, leading to features such as the infinite randomness fixed point and the associated quantum Griffiths phase anomalies [1, 2, 3, 4, 5, 6, 7, 8, 9]. Quantum critical regions, though, are much easier to access in itinerant systems such as heavy fermion materials, where quantum fluctuations can be tuned by adjusting the Fermi energy of the charge carriers.

In many of these systems, behavior reminiscent of quantum Griffiths phases seems to arise, triggering much theoretical controversy and debate. One early interpretation concentrated on the interplay of disorder and the Kondo effect far from any magnetic ordering, leading to the "electronic Griffiths phase" 10]. Another scenario assumed that the phenomenon relates to rare regions close to magnetic ordering [11], similar to those in insulating magnets. More recent work [12], however, shed some doubt on the validity of the latter picture for itinerant systems. It emphasized the crucial role of Landau damping, which was argued to suppress the low temperature Griffiths phase behavior for magnets with Ising symmetry. In a remarkable paper [13], Thomas Vojta discussed this mechanism on general symmetry grounds, and demonstrated that in the Ising case dissipation leads to a fundamental modification of the quantum critical point (QCP). Subsequent work [14] suggested that the same mechanism does not apply for other symmetry classes such as Heisenberg magnets, where the standard QCP scenario is argued to hold, preceded by a conventional Griffiths phase.

All these works concentrated on the dynamics of a single droplet (rare region) in an Ohmic dissipative environment. In this letter we argue that this physical picture is incomplete. In itinerant systems, magnetic moments - classical or quantum - are known [12, 16] to interact through long-ranged RKKY interactions. As a result,

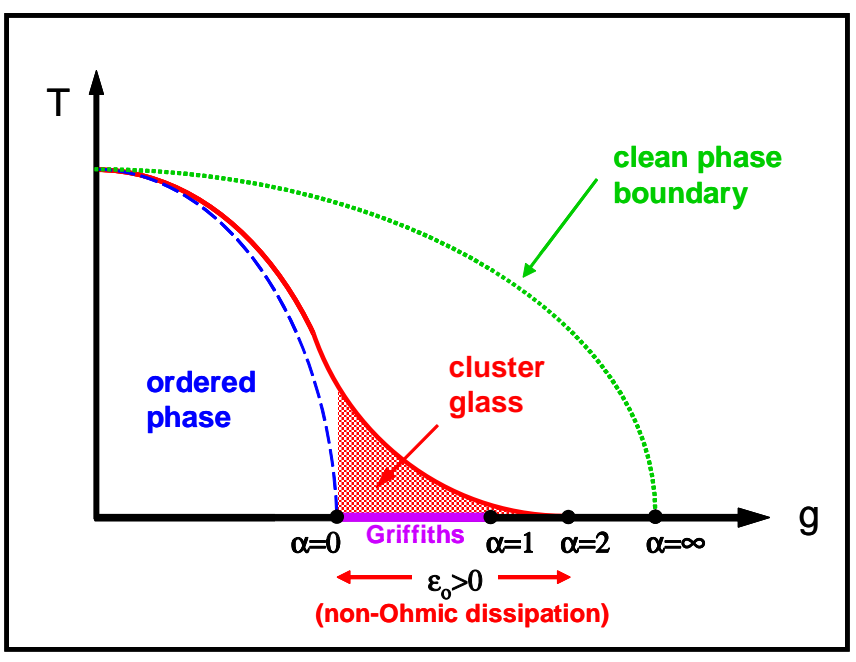

Figure 1: Phase diagram of an itinerant system with disorder, close to the clean quantum critical point (QCP). Shown are the clean critical line (dotted green) and that in the presence of disorder if non-Ohmic dissipation is ignored (dashed blue line). In this case, a Griffiths phase emerges close to the magnetically ordered phase, for $\alpha<1$ (purple). When dissipation is present, sufficiently large droplets "freeze" already for $\alpha<2$, leading to the formation of the "cluster glass" phase (shaded region), preceding the uniform ordering.

even the dynamics of spatially distant droplets cannot be considered as independent. The dynamics of any droplet is therefore affected not only by dissipation due to the conduction electrons in its vicinity, but also by spin fluctuations due to RKKY interactions with many other droplets. This effect is especially important in Griffiths phases where droplets are characterized by powerlaw distributions of local energy scales, with a tunable dynamical exponent $d / z^{\prime}$. In the rest of this letter we present a self-consistent theory describing the dynamics of interacting droplets. Within our theory: (1) dissipation produced by such spin fluctuations can acquire a more singular non-Ohmic form which can adversely affect 
the dynamics for all symmetry classes; (2) any Griffiths phase is destabilized by this mechanism, leading to the generic formation of a "cluster glass" phase preceding any uniform ordering, thus introducing a fundamental modification of the conventional QCP scenario.

Model of interacting droplets. To be specific, we concentrate on the same regime as explored in Ref. [14], and examine a weakly disordered itinerant antiferromagnet near a QCP. In the absence of disorder and within the standard Landau-Ginzburg-Wilson description, the distance to the QCP is measured by the coupling constant $r$. When disorder is present, exponentially rare regions (droplets) can arise which are nearly magnetically ordered. Within the $i$-th droplet of size $L_{i}$, the local value of the (bare) coupling constant is assumed to be $r_{i} \ll 0$, while outside the droplets (the "bulk") we have a weakly correlated metal $\left(r_{o} \gg 0\right)$. As discussed in Ref. 14], if the interactions between the droplets are ignored, then the dynamics of each droplet is determined only by its size. After appropriate coarse-graining, such a droplet is described by an action of the form

$$
\begin{aligned}
S_{d r o p}\left[r_{i}, \phi_{i}\right] & =\int_{o}^{\beta} d \tau d \tau^{\prime} \phi_{i}(\tau) \Gamma_{i}\left(\tau-\tau^{\prime}\right) \phi_{i}\left(\tau^{\prime}\right) \\
& +\frac{u}{2 N} \int_{o}^{\beta} d \tau \phi_{i}^{4}(\tau) .
\end{aligned}
$$

Here, $\phi_{i}$ is an $N$-component $(N>1)$ order parameter field describing the $i$-th droplet, and $\Gamma_{i}(\tau)$ is the coarsegrained two-point vertex whose Fourier transform is

$$
\Gamma_{i}\left(\omega_{n}\right)=\left(r_{i}+\left|\omega_{n}\right|\right) .
$$

The local "bare" coupling constant of each droplet $r_{i} \approx$ $r\left(L_{i}\right)^{d}$ reflects its size (here $r<0$ is the coupling constant of the clean magnet). Large droplets are exponentially rare (probability $P\left(L_{i}\right) \sim \exp \left\{-\rho\left(L_{i}\right)^{d}\right\}$ ), correspondingly $P\left(r_{i}\right) \sim \exp \left\{-\rho r_{i} / r\right\}$, where $\rho$ is the volume fraction of magnetic droplets . Now, each droplet maps onto a one-dimensional classical Heisenberg chain with $1 / \tau^{2}$ interactions and the corresponding energy gap $\varepsilon_{i} \sim \exp \left\{\pi r_{i} / u\right\}$ 13, 14]. The resulting distribution function $P\left(\varepsilon_{i}\right) \sim \varepsilon_{i}^{\alpha-1}$, giving rise to standard Griffiths phase behavior. The "Griffiths exponent" $\alpha=d / z^{\prime}$ is a nonuniversal function of parameters, which is expected 14] to decrease as the magnetic ordering is approached; for $\alpha<1$, the average susceptibility $\chi \sim T^{1-\alpha}$ diverges as $T \rightarrow 0$.

In contrast to the situation of Ref. [14], when a finite concentration of droplets is present, they will interact. The action describing the droplet-droplet interaction can be obtained by formally integrating over the order parameter fluctuations of the "bulk" separating the droplets. Using the fact that in this region the spin fluctuations are only weakly correlated $\left(r_{o} \gg 0\right)$, we can formally integrate out all the degrees of freedom in the bulk, to obtain an effective interaction coupling pairs [15] of droplets

$$
S_{i j}=\int_{o}^{\beta} d \tau d \tau^{\prime} \phi_{i}(\tau) \chi_{o}\left(R_{i j}, \tau-\tau^{\prime}\right) \phi_{j}\left(\tau^{\prime}\right),
$$

where $R_{i j} \gg L_{i}, L_{j}$ is the distance between droplets $i$ and $j$. The interaction kernel $\chi_{o}\left(R_{i j}, \tau-\tau^{\prime}\right)$ is, in fact, nothing but the non-local susceptibility of the weakly correlated "bulk" and as such can be accurately obtained from standard Fermi liquid considerations (see, e.g., [16]). Its detailed form is complicated and model-dependent, but for our problem only its long-distance and low frequency components are of importance, since the droplets are spatially distant and display slow dynamics. We can thus safely ignore its frequency dependence and consider only its asymptotic form, which is essentially that of the RKKY interaction

$$
\chi_{o}\left(R_{i j}, \tau-\tau^{\prime}\right) \approx \frac{J_{i j}}{\left(R_{i j}\right)^{d}} \delta\left(\tau-\tau^{\prime}\right) .
$$

In a disordered metal, impurity scattering introduces random phase fluctuations in the usual periodic oscillations of the RKKY interaction, which, however, retains its power law form (although its average value decays exponentially [16, 17]). Hence, such an interaction acquires a random amplitude $J_{i j}$ of zero mean and variance $<J_{i j}^{2}>=J^{2}$ [16, 17].

Field theory of interacting droplets. Because of the long-ranged interactions, one needs to describe the collective dynamics of the entire set of such droplets. To do this, we formally average over the random interactions $J_{i j}$ using standard replica methods, and the interaction term takes the form

$$
S_{i j} \approx-\frac{1}{2} \frac{J^{2}}{\left(R_{i j}\right)^{2 d}} \sum_{a, b=1}^{n} \int_{o}^{\beta} d \tau d \tau^{\prime} \phi_{i}^{a}(\tau) \phi_{i}^{b}\left(\tau^{\prime}\right) \phi_{j}^{a}(\tau) \phi_{j}^{b}\left(\tau^{\prime}\right),
$$

where $a, b=1, \ldots, n(n \rightarrow 0)$ are the replica indices. We then introduce collective $Q$-fields by decoupling $S_{i j}$ through a Hubbard-Stratonovich transformation to write the replicated partition function as

$$
Z^{n}=\int D \phi D Q \exp \left\{-S_{e f f}[\phi, Q]\right\},
$$

where the effective action

$$
\begin{aligned}
S_{\text {eff }}[\phi, Q] & =\frac{1}{2} \sum_{a, b=1}^{n} \sum_{i j, \omega_{n}} Q_{i}^{a b}\left(\omega_{n}\right) \frac{J^{2}}{\left(R_{i j}\right)^{2 d}} Q_{j}^{a b}\left(\omega_{n}\right) \\
& +\sum_{i}\left\{S_{d r o p}\left[r_{i}, \phi_{i}\right]+S_{M C}\left[\phi_{i}, Q\right]\right\},
\end{aligned}
$$

and the "mode-coupling" term

$$
S_{M C}\left[\phi_{i}, Q\right]=\sum_{a, b=1}^{n} \sum_{j, \omega_{n}} \phi_{i}^{a}\left(\omega_{n}\right) \phi_{i}^{b}\left(\omega_{n}\right) \frac{J^{2}}{\left(R_{i j}\right)^{2 d}} Q_{j}^{a b}\left(\omega_{n}\right) .
$$


Saddle point theory. Since the droplets are exponentially rare, their interactions are also very small. To describe the leading order corrections it is sufficient to evaluate the $Q$-fields at the saddle point level and the mode-coupling term assumes the form

$$
S_{M C}\left[\phi_{i}, \Delta\right]=-\sum_{a, b=1}^{n} \sum_{\omega_{n}} \phi_{i}^{a}\left(\omega_{n}\right) \Delta^{a b}\left(\omega_{n}\right) \phi_{i}^{b}\left(\omega_{n}\right),
$$

with the "cavity field" $\Delta^{a b}\left(\omega_{n}\right)$ satisfying the following self-consistency condition

$$
\Delta^{a b}\left(\omega_{n}\right)=\sum_{i} \frac{J^{2}}{\left(R_{i j}\right)^{2 d}}\left\langle\phi_{i}^{a}\left(\omega_{n}\right) \phi_{i}^{b}\left(\omega_{n}\right)\right\rangle_{i},
$$

where the expectation value is evaluated with respect to the local effective action $S_{l o c}\left[r_{i}, \phi_{i}\right]=S_{d r o p}\left[r_{i}, \phi_{i}\right]+$ $S_{M C}\left[\phi_{i}, \Delta\right]$. If all the droplets were the same, this set of equations would be identical to those describing a metallic quantum spin glass 18. For interactions $J$ sufficiently large, such a model would feature a conventional quantum critical point, where spin-glass ordering would set in at $T=0$. A crucial new feature in our model is a broad power-law distribution $P(\varepsilon) \sim \varepsilon^{\alpha-1}$ of local energy scales characterizing the droplets in a Griffiths phase, which produces non-Fermi liquid behavior for $\alpha<1$. We will see, however, that already for $\alpha<2$, qualitatively new dynamics emerges, completely changing the critical behavior of the model.

Instability of the Griffiths phase. Assuming that all droplets are well separated, the average interaction strength is very small. If we could ignore the fluctuations in the droplet sizes, the system would be far from any ordering. We thus concentrate on the paramagnetic phase of our model, where $\left\langle\phi_{i}^{a}\left(\omega_{n}\right) \phi_{i}^{b}\left(\omega_{n}\right)\right\rangle_{i}=\delta_{a b} \chi_{l o c}\left(\varepsilon_{i}, \omega_{n}\right)$. Since the droplet sizes (i.e. their local energy scales $\varepsilon_{i}$ ) are independent of their position, we find

$$
\Delta^{a b}\left(\omega_{n}\right)=\widetilde{g} \delta_{a b} \overline{\chi_{l o c}\left(\omega_{n}\right)}=\widetilde{g} \delta_{\alpha b} \int d \varepsilon_{i} P\left(\varepsilon_{i}\right) \chi_{l o c}\left(\varepsilon_{i}, \omega_{n}\right),
$$

where the RKKY coupling constant $\widetilde{g}=$ $J^{2} \sum_{i}\left(R_{i j}\right)^{-2 d} \sim n J^{2}$, where $n$ is the density of droplets per unit volume.

The "memory kernel" $\overline{\chi_{l o c}\left(\omega_{n}\right)}$ describes additional dissipation caused by spin fluctuations resulting from longranged RKKY interactions between the droplets. Its quantitative form is obtained by self-consistently solving the dynamics of each droplet in such a dissipative bath and then carrying out the appropriate averaging procedure. In this letter, however, we limit our attention to demonstrating the instability of the Griffiths phase with respect to adding the RKKY interactions. To do this, begin with $\widetilde{g}=0$ and imagine turning on infinitesimal
RKKY interactions. To leading order, we replace

$$
\begin{aligned}
\overline{\chi_{l o c}\left(\widetilde{g}, \omega_{n}\right)} & \approx \overline{\chi_{l o c}\left(0, \omega_{n}\right)} \sim \int d \varepsilon_{i} \varepsilon_{i}^{\alpha-1}\left[\varepsilon_{i}+\left|\omega_{n}\right|\right]^{-1} \\
& =\overline{\chi_{l o c}(0)}-\tilde{\gamma}\left|\omega_{n}\right|^{1-\varepsilon_{o}}+O\left(\left|\omega_{n}\right|\right),
\end{aligned}
$$

where the $\varepsilon_{o}=2-\alpha$, and $\tilde{\gamma}$ is a constant. Note that the dissipation acquires a non-Ohmic form for $\alpha<2$. As anticipated in Ref. [13], such non-Ohmic dissipation immediately changes the dynamics of the droplets. For $\varepsilon_{o}>0$, the Heisenberg chain [19] that represents the droplet dynamics (whose interactions decay with a power slower than $r^{-2}$ ) finds itself above its lower critical dimension! Accordingly, sufficiently large droplets $\left(L>L_{c}\left(\varepsilon_{o}\right)\right)$ "freeze", i.e. behave as classical (superparamagnetic) objects.

Cluster glass phase. As soon as some of the droplets freeze, they immediately order at a finite (albeit exponentially low) temperature, forming a "cluster glass" phase. To estimate the ordering temperature, we first need to determine the critical droplet size, given the anomalous dissipation exponent $\varepsilon_{o}$. For small values of $\varepsilon_{o}$, the corresponding critical exponents have been calculated [19] using an $\varepsilon$-expansion approach. For our purposes, it suffices to determine the "transition temperature" of the equivalent Heisenberg chain. This task is adequately accomplished using the large- $N$ approach. The corresponding $T=0$ self-consistency condition for the critical coupling constant reads ( $\Lambda$ is an ultraviolet cutoff)

$$
r_{i, c}=-u \int_{0}^{\Lambda} \frac{d \omega}{\pi} \frac{1}{\omega+\gamma \omega^{1-\varepsilon_{o}}},
$$

where $\gamma=\tilde{g} \tilde{\gamma}$. Note that for $\varepsilon_{o} \rightarrow 0$ or $\gamma \rightarrow 0$, the integral diverges in the infrared, indicating that $r_{i, c} \rightarrow-\infty$, i.e. that $L_{i, c} \rightarrow+\infty$. For $\varepsilon_{o}>0$, however, the term $\gamma \omega^{1-\varepsilon_{o}}$ introduces a soft cutoff regularizing the integral. This term dominates below a crossover frequency $\omega^{*}\left(\varepsilon_{0}\right)=\gamma^{1 / \varepsilon_{o}}$, yielding

$$
r_{i, c}\left(\varepsilon_{o}\right)=-\frac{u}{\pi \varepsilon_{o}} \ln (1 / \gamma) .
$$

The resulting concentration of frozen droplets is $n_{f r}\left(\varepsilon_{o}\right) \sim \exp \left\{-\rho r_{i, c}\left(\varepsilon_{o}\right) / r\right\}$. These frozen droplets produce a superparamagnetic contribution to the average susceptibility, proportional to their concentration $\delta \chi_{f r} \sim n_{f r}\left(\varepsilon_{o}\right) / T$. The ordering temperature can be immediately estimated using arguments similar to those of Ref. [13]. In particular, the RKKY interaction between droplets falls off as $J\left(R_{i j}\right) \sim R_{i j}^{-d}$ [20], where the typical distance between droplets $R_{i i} \sim\left[n_{f r}\left(\varepsilon_{o}\right)\right]^{-1 / d}$, and we obtain a simple exponential [21] dependence of $T_{c}$ on the dissipation exponent $\varepsilon_{o}$

$$
T_{c}\left(\varepsilon_{o}\right) \sim n_{f r}\left(\varepsilon_{o}\right) \sim \exp \left\{-\frac{\rho u}{\pi|r| \varepsilon_{o}} \ln (1 / \gamma)\right\} .
$$


Note that, within our model, the fraction of frozen droplets which magnetically order vanishes at a finite value of the coupling constant, producing a sharp phase transition from a quantum paramagnet to a cluster glass phase. In contrast to the infinite randomness fixed point (IRFP) scenario [2, 7, 9], this transition does not have any percolation-like features. In the itinerant case we consider, even very distant droplets will order as soon as they freeze, because they interact through long-ranged RKKY interactions. In this case, percolation processes [7, 9] associated with the IRFP scenario are not possible, suggesting that our phase transition is qualitatively different from those discussed in previous work.

We conclude that the standard QCP scenario is thus qualitatively modified by the dissipative effects, since an intermediate cluster glass phase generically emerges between the quantum disordered and any magnetically ordered phase in itinerant systems. It is easy to see that our conclusions also apply to models with arbitrary symmetry of the order parameter, since they all find themselves effectively above their lower critical dimension [13] due to the presence of non-Ohmic dissipation.

We should emphasize that our finite temperature ordering emerges over the entire region $\left(\alpha=d / z^{\prime}<1\right)$ where the Griffiths anomalies arise in the absence of RKKY interactions (see Fig. 1). Finite temperature ordering persists even further (until $\alpha=d / z^{\prime}=2$ ), but not all the way to the clean model critical point (corresponding to $\alpha=d / z^{\prime} \rightarrow+\infty$ according to the large$N$ estimates of Ref. [14]). Of course, since the droplets are diluted, the magnitude of the non-Ohmic dissipation produced by the RKKY interactions is expected to be considerably smaller then the standard Ohmic dissipation due to Landau damping. As a result, the effects of non-Ohmic dissipation that we predict should be seen only at fairly low temperatures $T<T^{*} \sim \omega^{*}=$ $\exp \left\{-\ln (1 / \gamma) / \varepsilon_{o}\right\}$, while the Griffiths phase behavior predicted by Ref. [14] should be observable in a broad interval above this temperature. Note that for $\rho u>\pi|r|$ (see Eq. [15), $T_{c}<<T^{*}$ for $\varepsilon_{o}<<1$, so there exists an appreciable temperature window where the the superparamagnetic contribution $\delta \chi_{f r} \sim 1 / T$ of frozen droplets should be observable. Otherwise $T_{c} \approx T^{*}$, and the Vojta-Schmalian Griffiths phase should emerge immediately above the ordering temperature. This scenario, valid for models with continuous symmetry of the order parameter, should be contrasted to that expected for the Ising universality class. In that special case, much stronger Ohmic dissipation is sufficient to suppress the low temperature Griffiths anomalies. For the Ising case, the existence of an intermediate temperature range where they would still be observable [11] remains the subject of some controversy [22].

In summary, we have established that the long-ranged part of the RKKY interaction between the droplets represents a singular perturbation within Griffiths phases.
Even when treated to leading order, the RKKY interactions qualitatively modify the droplet dynamics, leading to droplet freezing and suppression of conventional quantum critical behavior. Our arguments strongly suggest that the intervening cluster glass phase arises as a generic phenomenon [23] for itinerant systems with disorder. This mechanism is, however, specific to those quantum critical points associated with spontaneous symmetry breaking of a static order parameter. It would be very interesting to investigate if similar scenarios apply for other quantum critical points, such as the disorderdriven metal-insulator transition 24] and the associated electronic Griffiths phases [10, 24]. This fascinating direction remains a challenge for future work.

We acknowledge fruitful discussions with E. Abrahams, A. H. Castro Neto, A. J. Millis, D. K. Morr, J. Schmalian, S. Sachdev, T. Vojta, and K. Yang. This work was supported by the NSF through grant NSF-0234215 (V.D.), by FAPESP through grant 01/00719-8 (E.M.), by $\mathrm{CNPq}$ through grant 302535/02-0 (E.M.), and the National High Magnetic Field Laboratory (V.D. and E.M.). We also thank the Aspen Center for Physics, where part of this work was carried out.

[1] B. M. McCoy, Phys. Rev. Lett. 23, 383 (1969).

[2] D. S. Fisher, Phys. Rev. Lett. 69, 534 (1992); Phys. Rev. B 51, 6411 (1995).

[3] M. Thill and D. Huse, Physica A 214, 321 (1995).

[4] A. P. Young and H. Rieger, Phys. Rev. B 53, 8486 (1996).

[5] H. Rieger and A. P. Young, Phys. Rev. B 54, 3328 (1996).

[6] M. Guo et al., Phys. Rev. B 54, 3336 (1996).

[7] T. Senthil and S. Sachdev, Phys. Rev. Lett. 77, 5292 (1996).

[8] C. Pich et al., Phys. Rev. Lett. 81, 5916 (1998).

[9] O. Motrunich et al., Phys. Rev. B 61, 1160 (2000).

[10] D. Tanasković et al., cond-mat/0405005 E. Miranda and V. Dobrosavljević, Phys. Rev. Lett. 86, 264 (2001); E. Miranda et al., Phys. Rev. Lett. 78, 290 (1997).

[11] A. H. Castro Neto et al., Phys. Rev. Lett. 81, 3531 (1998); A. H. Castro Neto and B. A. Jones, Phys. Rev. B 62, 14975 (2000).

[12] A. J. Millis et al., Phys. Rev. Lett. 87, 167202 (2001); Phys. Rev. B 66, 174433 (2002).

[13] T. Vojta, Phys. Rev. Lett. 90, 107202 (2003).

[14] T. Vojta and J. Schmalian, cond-mat/0405609

[15] The formal procedure of integrating out the "bulk" separating droplets will, rigorously speaking, also generate effective interactions between three and more droplets. However, in the regime where the density of droplets is exponentially small, these contributions represent subleading terms, which can be safely neglected.

[16] B. Narozhny et al., Phys. Rev. B 62, 14898 (2000).

[17] A. Jagannathan et al., Phys. Rev. B 37, 436 (1988).

[18] S. Sachdev, Quantum Phase Transitions, (Cambridge University Press, 2001).

[19] J. M. Kosterlitz, Phys. Rev. Lett. 37, 1577 (1976).

[20] We ignore complications due to the finite temperature 
exponential cutoff of the RKKY power law.

[21] Ref. 13] suggested a double exponential form, in contrast to what we find. This follows from assuming that the droplet-droplet interactions decrease exponentially with distance, as one expects for insulating magnets in a paramagnetic phase. For itinerant systems, in contrast, the magnetic RKKY interactions are generically long ranged, giving rise to a simple exponential dependence.

[22] A. H. Castro Neto and B. A. Jones, cond-mat/0411197 and cond-mat/0412020 A. J. Millis, D. K. Morr, and J. Schmalian, cond-mat/0411738

[23] The droplet arguments that we and others have used to discuss the Griffiths phase behavior in principle apply in all spatial dimensions. Our self-consistent approach to RKKY interactions, however, is rigorously justified only for sufficiently large dimensions. The role of spatial fluctuations in low dimensions and the possible modifications of our scenario in this limit are subtle questions that require further work.

[24] V. Dobrosavljević and G. Kotliar, Phys. Rev. Lett. 78, 3943 (1997); V. Dobrosavljević et al., Phys. Rev. Lett. 90, 016402 (2003). 